\title{
Quantitative T1 Mapping for Detecting Microvascular Obstruction in Reperfused Acute Myocardial Infarction: Comparison with Late Gadolinium Enhancement Imaging
}

\author{
Jae Min Shin, $\mathrm{MD}^{1}$, Eui-Young Choi, MD, PhD², Chul Hwan Park, MD, PhD ${ }^{1}$, Kyunghwa Han, PhD ${ }^{3}$, \\ Tae Hoon Kim, MD, PhD ${ }^{1}$
}

\begin{abstract}
${ }^{1}$ Department of Radiology and the Research Institute of Radiological Science, Gangnam Severance Hospital, Yonsei University College of Medicine, Seoul, Korea; ${ }^{2}$ Division of Cardiology, Heart Center, Gangnam Severance Hospital, Yonsei University College of Medicine, Seoul, Korea; ${ }^{3}$ Department of Radiology and the Research Institute of Radiological Science, Severance Hospital, Yonsei University College of Medicine, Seoul, Korea
\end{abstract}

Objective: To compare native and post-contrast T1 mapping with late gadolinium enhancement (LGE) imaging for detecting and measuring the microvascular obstruction (MVO) area in reperfused acute myocardial infarction (MI).

Materials and Methods: This study included 20 patients with acute MI who had undergone 1.5T cardiovascular magnetic resonance imaging (CMR) after reperfusion therapy. CMR included cine imaging, LGE, and T1 mapping (modified look-locker inversion recovery). MI size was calculated from LGE by full-width at half-maximum technique. MVO was defined as an area with low signal intensity (LGE) or as a region of visually distinguishable T1 values (T1 maps) within infarcted myocardium. Regional T1 values were measured in MV0, infarcted, and remote myocardium on T1 maps. MVO area was measured on and compared among LGE, native, and post-contrast T1 maps.

Results: The mean MI size was $27.1 \pm 9.7 \%$ of the left ventricular mass. Of the 20 identified MV0s, 18 (90\%) were detected on native T1 maps, while $10(50 \%)$ were recognized on post-contrast T1 maps. The mean native T1 values of MV0, infarcted, and remote myocardium were 1013.5 $\pm 58.5,1240.9 \pm 55.8(p<0.001)$, and $1062.2 \pm 55.8 \mathrm{~ms}(p=0.169)$, respectively, while the mean post-contrast T1 values were $466.7 \pm 26.8,399.1 \pm 21.3$, and $585.2 \pm 21.3 \mathrm{~ms}$, respectively $(p<0.001)$. The mean MVO areas on LGE, native, and post-contrast T1 maps were $134.1 \pm 81.2,133.7 \pm 80.4$, and $117.1 \pm 53.3 \mathrm{~mm}^{2}$, respectively. The median (interquartile range) MVO areas on LGE, native, and post-contrast T1 maps were 128.0 (58.1-215.4), 110.5 (67.7-227.9), and $143.0(76.7-155.3) \mathrm{mm}^{2}$, respectively $(p=0.002)$. Concordance correlation coefficients for the MV0 area between LGE and native T1 maps, LGE and post-contrast T1 maps, and native and post-contrast T1 maps were 0.770, 0.375, and 0.565 , respectively.

Conclusion: MV0 areas were accurately delineated on native T1 maps and showed high concordance with the areas measured on LGE. However, post-contrast T1 maps had low detection rates and underestimated MV0 areas. Collectively, native T1 mapping is a useful tool for detecting MVO within the infarcted myocardium.

Keywords: Microvascular obstruction; Myocardial infarction; T1 mapping; Late gadolinium enhancing imaging; Cardiovascular magnetic resonance imaging

\section{INTRODUCTION}

Cardiovascular magnetic resonance imaging (CMR) is currently the reference standard for evaluating cardiac structure and function in acute coronary syndromes. Late gadolinium enhancement (LGE) imaging is used very widely

Received: October 5, 2019 Revised: February 15, 2020 Accepted: March 5, 2020

This study was partially supported by research grant from Dong-A ST (3-2011-0203).

Corresponding author: Tae Hoon Kim, MD, PhD, Department of Radiology, Gangnam Severance Hospital, 211 Eonju-ro, Gangnam-gu, Seoul 06273, Korea.

- E-mail: thkim1@yuhs.ac

This is an Open Access article distributed under the terms of the Creative Commons Attribution Non-Commercial License (https:// creativecommons.org/licenses/by-nc/4.0) which permits unrestricted non-commercial use, distribution, and reproduction in any medium, provided the original work is properly cited. 
in contemporary clinical practice and cardiac imaging and is considered the gold standard for assessing myocardial viability and for differentiating ischemic and non-ischemic cardiomyopathy (1-3). Along with its diagnostic utility for acute myocardial infarction (MI), LGE imaging is also useful for detecting microvascular obstruction (MVO), an important prognostic factor of poor outcomes in patients with MI (4-6). MV0 generally occurs following reperfusion therapy after prolonged myocardial ischemia and is histologically associated with hemorrhagic infarction. It results from luminal narrowing of the myocardial microvasculature caused by ischemic endothelial injury, and aggregates of fibrin, platelets, and erythrocytes then obstruct the narrowed microvasculature. This is known as the 'no-reflow' phenomenon $(4,7)$.

On LGE images, MVO presents as a dark non-enhanced area within the enhanced infarcted myocardium. Patients with MVO on LGE images usually demonstrate adverse left ventricular remodeling, resulting in subsequent major cardiovascular events and even death $(4,7)$. Since LGE imaging is now widely employed, a gadolinium-based contrast agent (GBCA) is currently administered in $>80 \%$ of CMR procedures (3). However, caution is needed when using a GBCA, because the clinical significance of its tissue deposition remains unclear $(3,8)$.

T1 mapping is a recently developed technique that measures the $\mathrm{T} 1$ relaxation time in pixels directly from the affected myocardium, without the need for GBCA administration $(1,9,10)$. The $T 1$ relaxation time of the myocardium depends on the disease state, due to changes in the tissue microenvironment (9). In MI, T1 values reportedly increase owing to ischemia and edema $(1,11)$. Nevertheless, a few studies have detected MVO using T1 mapping $(1,11)$. The presence of MVO is thought to decrease T1 values due to the extravasation of iron-containing erythrocytes and myocardial hemorrhage in MVO $(1,4,11)$. Post-contrast T1 mapping is also valuable for tissue characterization, and measuring $\mathrm{T} 1$ values with extracellular GBCA provides information on the extracellular volume (ECV) fraction $(12,13)$. However, the injected GBCA can produce many confounding factors and the images are not based solely on $\mathrm{T} 1$ values $(5,13)$. To the best of our knowledge, this is the first study to focus specifically on the detection of MVO using both native and post-contrast T1 mapping images.

Therefore, we aimed to evaluate the usefulness of T1 mapping for detecting MVO in reperfused acute MI and to compare measured MVO areas among native T1 maps, post- contrast T1 maps, and LGE images.

\section{MATERIALS AND METHODS}

\section{Study Population}

This retrospective and observational study was approved by the Institutional Review Board of Gangnam Severance Hospital (IRB No. 3-2019-0061). The requirement for informed consent was waived, because all images and clinical data were retrospectively obtained from medical records.

In our institute, the database of patients who underwent CMR has been maintained from January 2014 and contained 267 patients by December 2018. We retrospectively reviewed the database and found 78 patients with acute MI (ST-segment elevation MI [STEMI], $\mathrm{n}=50$; non STEMI, $\mathrm{n}=28$ ) who had undergone primary percutaneous coronary intervention (PCI) and then performed CMR. Among them, 20 patients were mentioned by MVO on the LGE images from CMR reports. Those 20 patients (all men; mean age: $54.3 \pm$ 11.8 years) were included. All CMR was performed within 7 days of admission, including LGE, native T1 mapping, and post-contrast $\mathrm{T} 1$ mapping sequences. The mean interval between PCI and CMR was $3.3 \pm 2.2$ days.

\section{Image Acquisition}

All CMR scans were performed with a 1.5-Tesla scanner (Magnetom Avanto, Siemens Healthineers, Erlangen, Germany) using a 12-element phased-array coil. Heart localization was performed using true fast imaging with steady-state precession (True FISP, Siemens Healthineers) under electrocardiographic gating. The protocols included cine CMR, native T1 mapping, LGE imaging, and postcontrast T1 mapping. Cine images were acquired using a steady-state free precession sequence in the short axis and 2-, 3-, and 4-chamber views of the left ventricle. The imaging parameters were as follows: repetition time (TR), $2.63 \mathrm{~ms}$; echo time (TE), $1.1 \mathrm{~ms}$; flip angle, $67^{\circ} ; 25$ phases; slice thickness, $8 \mathrm{~mm}$; slice gap, $2 \mathrm{~mm}$; acquisition matrix, $192 \times 109$; and field-of-view (FOV), $320 \times 400$ mm. Native T1 mapping was performed using a modified look-locker inversion recovery (MOLLI) sequence with a 5(3)3 protocol (14) that acquires 5 images after the first inversion pulse, followed by 3 RR intervals for T1 recovery, and a second inversion pulse after which 3 more images are acquired. Eight slices of T1 maps were obtained to cover the left ventricle fully. The imaging parameters were as follows: TR, 
$740 \mathrm{~ms}$; TE, $1.06 \mathrm{~ms}$; flip angle, $35^{\circ}$; slice thickness, $8 \mathrm{~mm}$; acquisition matrix, $192 \times 124$; and FOV, $320 \times 400$ mm (14).

LGE imaging, with a magnitude- and phase-sensitive inversion recovery prepared fast gradient echo sequence, was conducted 10 minutes after injecting a $0.2 \mathrm{mmol} / \mathrm{kg}$ intravenous dose of gadoterate dimeglumine (Dotarem, Guerbet, Roissy, France). A bolus of contrast media was intravenously administered at $2 \mathrm{~mL} / \mathrm{s}$, followed by $20 \mathrm{~mL}$ of normal saline at $4 \mathrm{~mL} / \mathrm{s}$ through a 20 -gauge cannula in the antecubital vein using a power injector (Nemoto, Nemoto Kyorindo, Tokyo, Japan). The appropriate inversion time was determined before LGE imaging using a fast gradient echo sequence with inversion times varying from 150 to $650 \mathrm{~ms}$ to null the signal from the normal myocardium. The LGE imaging parameters were as follows: TR, $8.8 \mathrm{~ms}$; $\mathrm{TE}, 3.36 \mathrm{~ms}$; flip angle $25^{\circ}$; acquisition matrix, $256 \mathrm{x}$ 166; and FOV, $276 \times 340 \mathrm{~mm}$. Post-contrast T1 maps were also acquired using the MOLLI sequence, with a 4(1)3(1)2 scheme (14), 15 minutes after contrast agent injection. In addition, eight slices of T1 maps were obtained to cover the left ventricle fully. The imaging parameters were the same as those used for native T1 mapping.

\section{Image Analysis}

Two radiologists (who had more than 10 years of experience in the field of cardiothoracic radiology) performed independent assessments, including qualitative and quantitative analyses, of all images. The patient's identity was blinded and the patient's orders were random. The two radiologists simultaneously reviewed three sets of images (LGE, native T1 map, and post-contrast T1 map) of the same patient. The radiologists were aware that three sets of images were from the same patient. The copresented LGE image was used as the reference to detect the infarcted myocardium and MVO on T1 maps.

Myocardial injury in ischemic conditions was defined as a visually identified region of hyper-enhancement in the left ventricular myocardium on LGE images. The MI size was calculated from the LGE images by the full-width at halfmaximum technique. MVO was defined separately according to the imaging sequence used. On LGE images, MVO was defined as a hypo-enhanced region within a hyper-enhanced infarcted myocardium. On native and post-contrast T1 maps, MV0 was defined as a region of visually distinguishable T1 values within the infarcted myocardium. If the MVO could be identified, the MVO area was measured by manually drawing its margin using a picture archiving and communication system station (Centricity 4.0, GE Healthcare, Chicago, IL, USA). The measured MVO areas on native and post-contrast T1 maps were compared with those on LGE images. The MVO lesions on LGE images, which were matched to the MVO lesions, and visualized on the native T1 and post-contrast T1 maps were categorized separately and the mean areas were calculated. In addition, the T1 value was measured by the MVO contour and the circles in the infarcted and remote myocardium on the native and post-contrast T1 maps using the picture archiving and communication system station. Three T1 values on each T1 map were compared.

\section{Statistical Analysis}

Categorical variables are expressed as frequencies (percentage) and continuous variables are presented as the mean \pm standard deviation (SD) for normally distributed variables and as the median (interquartile range [IQR]) for non-normally distributed variables. Normality assumptions for continuous variables were confirmed with the ShapiroWilk test.

A linear mixed model with Bonferroni correction was used to identify differences among the mean $\mathrm{T} 1$ values of the MVO, infarcted myocardium, and remote myocardium on native and post-contrast T1 maps. The median MV0 areas on LGE images, native T1 maps, and post-contrast T1 maps were compared using the Friedman test. For post-hoc pairwise multiple comparisons, Conover's test was performed. Differences were considered statistically significant at $p<0.05$.

The concordance correlation coefficient (CCC) was used to evaluate the reproducibility of measuring the MVO area on LGE images, native T1 maps, and post-contrast T1 maps. Inter-observer variability with respect to measuring the MVO area and T1 values was assessed with the intraclass correlation coefficient (ICC). The agreement levels were as follows: poor, ICC $<0.40$; fair to good, ICC $=0.40-0.75$; and excellent, $\mathrm{ICC}=0.76-1.00$. A Bland-Altman analysis was conducted to investigate the agreement of the MVO area measurements among the imaging methods. Statistical analyses were performed using commercially available software (SPSS for Windows version 23.0, IBM Corp., Armonk, NY, USA; MedCalc version 11.4, MedCalc Software bvba, Mariakerke, Belgium).

\section{RESULTS}

The characteristics and risk factors of the 20 patients 
are summarized in Table 1. Of the 20 patients with MVO detected on LGE images, MVO was visualized in 18 patients $(90 \%)$ on native T1 maps and in 10 patients $(50 \%)$ on postcontrast T1 maps. In most cases, except false negatives, the MVO area was well delineated within the infarcted myocardium on LGE images, native T1 maps, and postcontrast T1 maps (Fig. 1). The mean MVO areas on LGE images, native T1 maps, and post-contrast T1 maps were

Table 1. Characteristics of 20 Patients with Acute Myocardial Infarction Who Underwent Reperfusion Treatment

\begin{tabular}{lc}
\hline \multicolumn{1}{c}{ Parameter } & Total $(\mathrm{n}=20)$ \\
\hline Age, years & $54.3 \pm 11.8$ \\
Male/female, number $(\%)$ & $20(100) / 0(0)$ \\
Body mass index, $\mathrm{kg} / \mathrm{m}^{2}$ & $23.7 \pm 3.6$ \\
Hypertension, number (\%) & $11(55)$ \\
Hyperlipidemia, number (\%) & $6(30)$ \\
$\begin{array}{l}\text { Smoking history: current/ex-smoker/ } \\
\text { never, } \mathrm{n}(\%)\end{array}$ & $0(55) / 6(30) / 3(15)$ \\
$\begin{array}{l}\text { Diabetes, } \mathrm{n}(\%) \\
\text { STEMI/NSTEMI, number (\%) }\end{array}$ & $19(95) / 1(5)$ \\
Peak troponin T (median \pm IQR), $\mu \mathrm{g} / \mathrm{L}$ & $42.1 \pm 41.0$ \\
Time from onset of symptoms to PCI, & $3.4 \pm 1.4$ \\
hours & $1.8 \pm 0.9$ \\
Time from presentation to hospital to & $3.3 \pm 2.2$ \\
PCI, hours & $27.1 \pm 9.7$ \\
Time from PCI to CMR, days & \\
Mean size of myocardial infarction, \\
$\%$ of left ventricular mass
\end{tabular}

Data are presented as mean \pm standard deviation unless otherwise noted. $\mathrm{CMR}=$ cardiovascular magnetic resonance imaging, $\mathrm{IQR}=$ interquartile range, NSTEMI = non-ST-segment elevation myocardial infarction, $\mathrm{PCI}=$ percutaneous coronary intervention, $\mathrm{STEMI}=\mathrm{ST}$ segment elevation myocardial infarction
$134.1 \pm 81.2 \mathrm{~mm}^{2}, 133.7 \pm 80.4 \mathrm{~mm}^{2}$, and $117.1 \pm 53.3 \mathrm{~mm}^{2}$, respectively. The two MVO lesions not visualized on native T1 maps had areas measuring $30.4 \mathrm{~mm}^{2}$ and $44.4 \mathrm{~mm}^{2}$ and were the two smallest MVO lesions noted on LGE images. All MVO areas measured on post-contrast T1 maps were smaller than the matched MVO areas measured on LGE images $(p=0.027)$.

The median (IQR) MVO areas on LGE images $(n=20)$, native T1 maps $(n=18)$, and post-contrast T1 maps $(n=10)$ were $128.0(58.1-215.4) \mathrm{mm}^{2}, 110.5(67.7-227.9) \mathrm{mm}^{2}$, and $143.0(76.7-155.3) \mathrm{mm}^{2}$, respectively, and were significantly different among them $(p=0.002)$ (Fig. 2). However, pairwise comparisons of the MVO areas between the groups did not show any significant differences (Table 2). The CCCs for the measured MVO areas between image types are shown in Table 2. Agreement was relatively high between LGE images and native T1 maps $(n=18, C C C=0.770)$. Agreement was not good between LGE images and postcontrast T1 maps $(n=10, C C C=0.375)$. The mean area of the 10 MVO lesions detected on the post-contrast T1 maps was measured as $190.6 \pm 69.7 \mathrm{~mm}^{2}$, much larger than that of all 20 MVO lesions on the LGE images $(117.1 \pm 53.3$ $\left.\mathrm{mm}^{2}\right)$. The Bland-Altman analysis showed that the mean difference in MVO area was $9.8 \%(n=18,95 \%$ confidence interval [CI]: $-11.5-31.0 \%$ ) between LGE images and native T1 maps, with a coefficient of repeatability (2 SDs) of $83.8 \%$. The mean difference in MVO area was $51.9 \%$ ( $n=10,95 \%$ CI: $22.2-81.6 \%$ ) between LGE images and post-contrast $\mathrm{T} 1$ maps, with a coefficient of repeatability (2 SDs) of $81.4 \%$ (Fig. 3 ).

The mean T1 values of infarcted myocardium, MV0, and remote myocardium on native T1 maps $(n=18)$ were

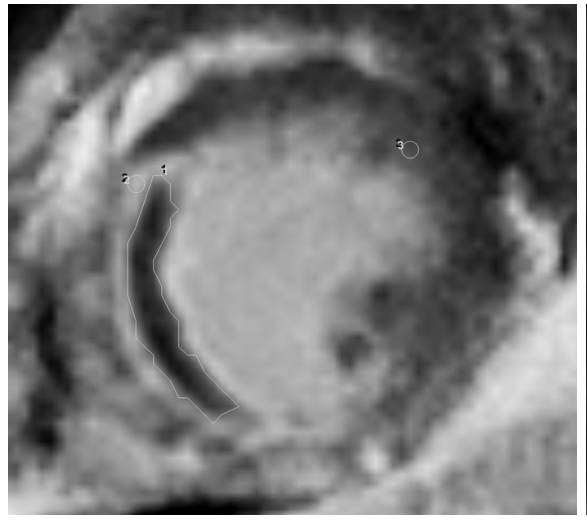

A

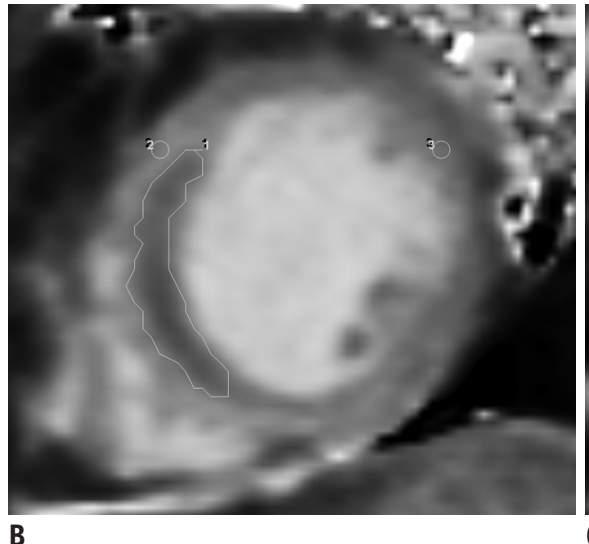

B

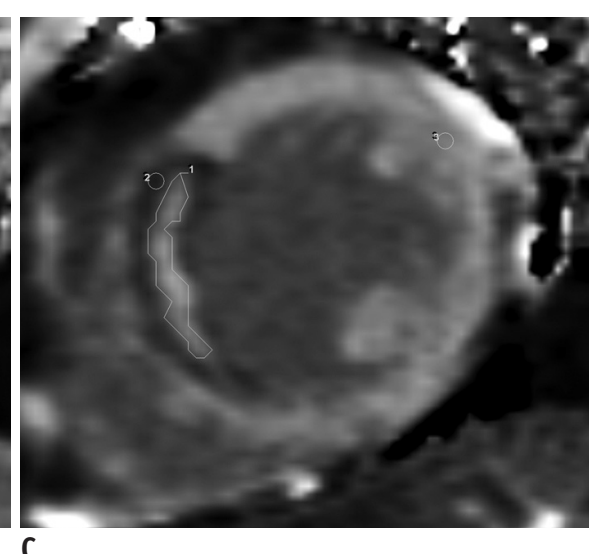

c

Fig. 1. MVO within infarcted myocardium on LGE image, native T1 map, and post-contrast T1 map.

A. On LGE image, MVO is observed as hypo-enhanced region within hyper-enhanced infarcted myocardium. B. On native T1 map, MVO is distinguished as dark region within bright infarcted myocardium. C. On post-contrast T1 map, MV0 is identified as bright region within dark infarcted myocardium. Regions of interest, in which T1 values were measured in (B) and (C), were outlined with circles in infarcted and remote myocardium and with contour of MVO. LGE = late gadolinium enhancement, MVO = microvascular obstruction 
$1240.9 \pm 55.8 \mathrm{~ms}, 1013.5 \pm 58.5 \mathrm{~ms}$, and $1062.2 \pm 55.8 \mathrm{~ms}$, respectively (Fig. $4 \mathrm{~A}$ ). On native $\mathrm{T} 1$ maps, the mean T1 value of the MVO was significantly lower than that of the infarcted myocardium $(p<0.001)$ and slightly, but not significantly, lower than that of the remote myocardium $(p=0.169)$. The mean T1 values of infarcted myocardium, MVO, and remote myocardium on post-contrast T1 maps $(n=10)$ were $399.1 \pm$ $21.3 \mathrm{~ms}, 466.7 \pm 26.8 \mathrm{~ms}$, and $585.2 \pm 21.3 \mathrm{~ms}$, respectively (Fig. 4B). On post-contrast T1 maps, the mean T1 value of the MVO was significantly higher than that of the infarcted myocardium $(p<0.001)$ and significantly lower than that of the remote myocardium $(p<0.001)$.

A complete agreement has been reached on MVO detection by two radiologists. The inter-observer variability in measuring the MVO area on LGE images, native T1 maps, and post-contrast T1 maps was excellent, with ICCs of 0.984, 0.977 , and 0.969 , respectively. The inter-observer variability in measuring the $\mathrm{T} 1$ values on native T1 maps was excellent (ICC, 0.840), while that for post-contrast T1 maps was good (ICC, 0.696).

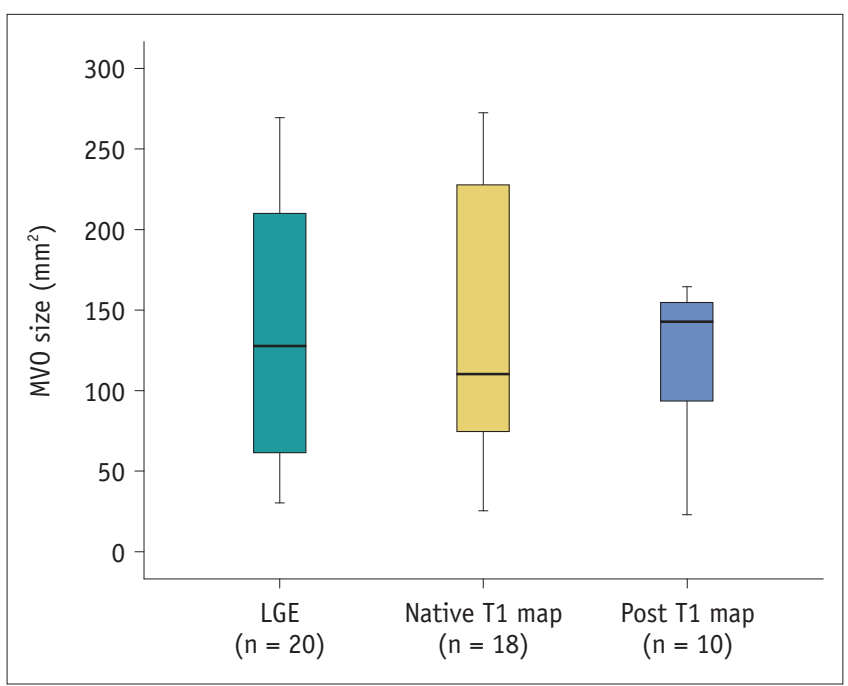

Fig. 2. MVO areas in 20 patients on LGE images, 18 patients on native $\mathrm{T} 1$ maps, and 10 patients on post-contrast T1 maps. Boxes, 1st-3rd quartiles; bold lines, medians; whiskers, minimum and maximum values.

\section{DISCUSSION}

Herein, we compared native and post-contrast T1 mapping with LGE imaging for delineating the MVO area in reperfused acute MI. There were three main findings. First, MVO was detected on native T1 maps in $90 \%$ of cases, but only detected in $50 \%$ of the cases on post-contrast $\mathrm{T} 1$ maps. Post-contrast T1 maps showed tendency to detect large MVO lesions, rather than small ones. Second, the MV0 area was accurately delineated and measured on native $\mathrm{T} 1$ maps and the measured MVO areas were highly concordant with those measured on LGE images. On native T1 maps, the mean T1 value of the MVO was lower than that of the infarcted myocardium but not significantly different from that of the remote myocardium. Third, MVO areas measured on post-contrast $\mathrm{T} 1$ maps were underestimated relative to those measured on LGE images. On post-contrast T1 maps, the mean T1 value of the MVO was higher than that of the infarcted myocardium but lower than that of the remote myocardium.

Many studies have compared native T1 maps with LGE or T2-weighted images in terms of their ability to detect MI (2, $9-11,15,16)$. However, a few studies have compared the utility of native or post-contrast T1 maps with that of LGE images for evaluating MVO (17-19). T1 maps can be used to objectively measure and directly quantify the absolute $\mathrm{T} 1$ relaxation time of the myocardium without contrast medium, thus providing an alternative imaging approach for patients with renal impairment. Moreover, the recent development and validation of a new native T1 mapping sequence (shortened MOLLI) allows in vivo T1 measurements and T1 mapping of the myocardium with a higher spatial resolution within one breath-hold $(9,20)$. In this study, native T1 maps exhibited sensitive delineation of MVO and yielded accurate area measurements. The mean MVO area measured on native T1 maps was not significantly different from that measured on LGE images and the reproducibility of MVO area measurements between these image types was high.

In contrast, on post-contrast T1 maps, the MV0 detection

Table 2. Pairwise Comparisons of Measured MVO Areas between Different Image Types and CCCs for MV0 Areas Measured between Different Image Types

\begin{tabular}{lccc}
\hline $\begin{array}{c}\text { Pairwise } \\
\text { Comparisons }\end{array}$ & $\begin{array}{c}\text { LGE vs. Native T1 Map } \\
(\mathrm{n}=18)\end{array}$ & $\begin{array}{c}\text { LGE vs. Post-Contrast T1 Map } \\
(\mathrm{n}=10)\end{array}$ & $\begin{array}{c}\text { Native T1 Map vs. Post-Contrast T1 Map } \\
(\mathrm{n}=10)\end{array}$ \\
\hline $\begin{array}{l}p \text { value } \\
\text { CCC }(95 \% \mathrm{CI})\end{array}$ & 0.69 & 0.69 & 0.43 \\
\hline
\end{tabular}

$\mathrm{CCC}=$ concordance correlation coefficient, $\mathrm{CI}=$ confidence interval, $\mathrm{LGE}=$ late gadolinium enhancement, $\mathrm{MVO}=$ microvascular obstruction 


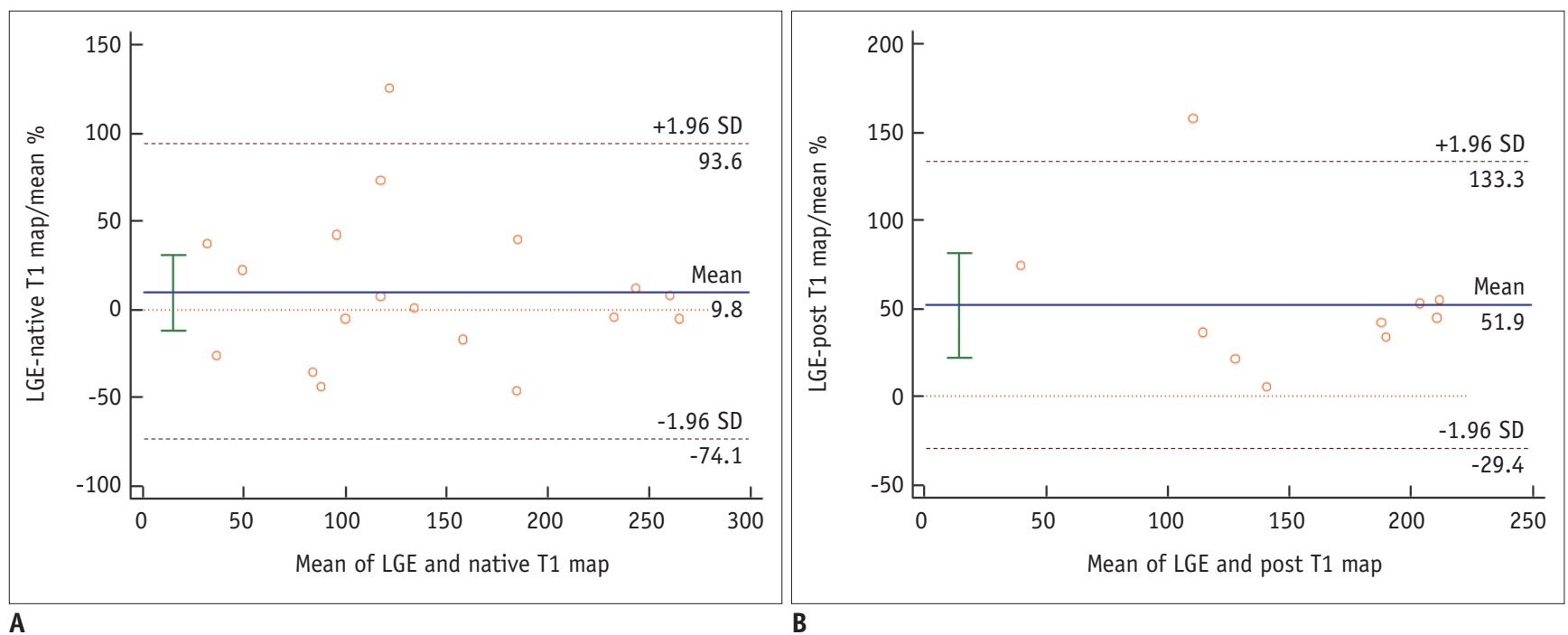

Fig. 3. Bland-Altman plots showing comparison of MVO areas between image types.

A. Mean difference in MVO area between LGE images and native T1 maps was 9.8\% ( $n=18,95 \%$ CI: $-11.5-31.0 \%$, and coefficient of repeatability (2 SDs) was $83.8 \%$. B. Mean difference in MVO size between LGE images and post-contrast T1 map images was $51.9 \%(n=10,95 \%$ CI: $22.2-81.6 \%$ ), and coefficient of repeatability (2 SDs) was $81.4 \%$. CI = confidence interval, SD = standard deviation

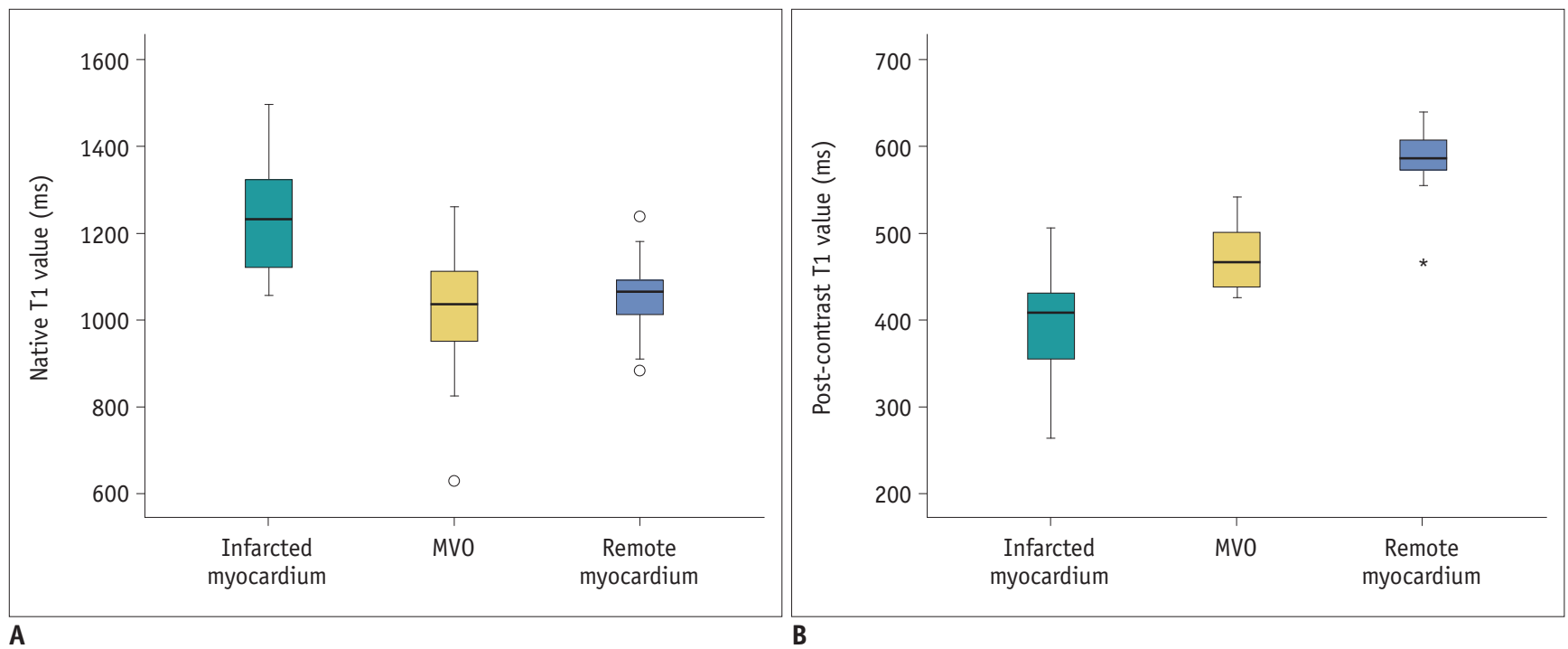

Fig. 4. T1 values of infarcted myocardium, MV0, and remote myocardium on native T1 maps $(n=18)$ and post-contrast T1 maps $(n=10)$.

A. On native T1 maps, mean T1 value of MVO was lower than that of infarcted myocardium, but was not statistically different from that of remote myocardium. B. On post-contrast T1 maps, mean T1 value of MVO was significantly higher than that of infarcted myocardium, but significantly lower than that of remote myocardium. Boxes, 1st-3rd quartiles; bold lines, medians; whiskers, minimum and maximum values; 0, outliers. *outliers.

rate was only $50 \%$ and all detected MVO areas were underestimated compared to those on LGE images. Ten relatively larger MVO lesions measured on LGE images (mean area on LGE, $190.6 \pm 69.7 \mathrm{~mm}^{2}$ ) were detectable on postcontrast T1 maps, compared to the other 10 lesions (mean area on LGE, $77.6 \pm 44.5 \mathrm{~mm}^{2}$ ). Many confounding factors in the post-contrast $\mathrm{T} 1$ measurements may have contributed to this underestimation, including the contrast dose, contrast clearance rate, scan timing, body composition, and hematocrit $(3,13)$. Because of these confounders, significant variations in the post-contrast $\mathrm{T} 1$ maps are inevitable. Mather et al. (18) reported that the MVO extent on first-pass perfusion images (median mass: $4.7 \mathrm{~g}$ ) was greater than those on early GBCA enhancement images (median mass: $2.3 \mathrm{~g}, p<0.05$ ) and LGE images (median mass: $0.2 \mathrm{~g}, p<0.05)$ in 34 patients with acute STEMI 
treated with primary PCI. Several studies, including that by Mather et al. (18), showed that GBCA extravasated into the interstitium and passively diffused into the MVO area $(21,22)$. Therefore, the MVO area decreased over time following GBCA injection. The LGE images and post-contrast T1 maps in the present study were obtained 10 minutes and 15 minutes after GBCA injection, respectively. Thus, based on the abovementioned previous studies, post-contrast T1 maps may underestimate the MVO area relative to LGE images because there are five more minutes for GBCA to diffuse into the MVO area. Although the post-contrast T1 mapping did not demonstrate the preferred output in this study, it provides important information on the ECV fraction or myocardial ECV, which is altered by infiltrative myocardial disease, edema, fibrosis, or myocardial remodeling (23).

In this study, the mean native T1 relaxation time of MVO was significantly lower than that of infarcted myocardium without MV0, and this finding is similar to the results of previous studies $(1,5,9,10)$. Prior studies using histological assessments revealed that the segments of acute MI demonstrate increased tissue water content and that such segments show increased T1 values on native T1 maps $(9,24)$. Edema is a typical feature of ischemiareperfusion injury and represents the inflammation state of the infarcted myocardial tissues $(9,25)$. Myocardial hemorrhage is another component of reperfusion injury that results from the extravasation of red blood cells through damaged endothelial walls during reperfusion (1, $9,10,22)$. Hemorrhage is frequently visible within the infarct core and is closely related to MVO. Hemoglobin in the extravasated red blood cells undergoes oxidative denaturation and produces paramagnetic substances, including deoxyhemoglobin, methemoglobin, ferritin, and hemosiderin, which result in shortening of the native $\mathrm{T} 1$ values of MVOs $(10,25-27)$. Moreover, the reduced water content in the MVO contributes to the shortening of the native $T 1$ values compared to the infarcted tissues without MVO $(9,28,29)$.

In previous studies, the mean native $\mathrm{T} 1$ value of the MV0 was higher than that of the remote myocardium $(1,5,9,10)$. However, in our study, the mean native T1 value of the MVO was lower, though not significantly, than that of the remote myocardium. Biesbroek et al. (5) revealed that patients with more extensive myocardial damage demonstrate higher native $\mathrm{T} 1$ values at the remote myocardium after treatment with primary PCI. In that study, the median infarct size measured on LGE images was $16 \%$
(IQR: $8-26 \%$ ) of the left ventricular mass and $55 \%$ of the patients had MVO (5). These changes might be associated with adverse cardiac remodeling after acute MI, and the severity of myocardial damage likely determined the tissue response of the remote myocardium. The mean infarct size measured on LGE images in our study was $27.1 \pm 9.7 \%$ of the left ventricular mass and the median infarct size was $24 \%(18-36 \%)$ of the left ventricular mass, which is larger than that in the previous study (5). Furthermore, MV0 was detected in all 20 patients in this study on LGE images. Hence, extensive myocardial damage may explain why the mean native $T 1$ value of the remote myocardium was higher than that of MVO in our study.

On post-contrast T1 maps, the mean T1 value of the MVO was significantly higher than that of the infarcted myocardium, but significantly lower than that of the remote myocardium. This is because the GBCA stayed in the infarcted tissues, causing shortening of the $\mathrm{T} 1$ relaxation time. Similar to native T1 maps, myocardial hemorrhage may have contributed to the lower T1 value in the MVO than in the remote myocardium for post-contrast T1 maps.

There are several limitations to this study. First, MV0 detection and area measurements were assessed visually. Many artifacts, including partial volume effects, cardiac and respiratory motion artifacts, B0-field inhomogeneity, and edge artifacts may have interfered with accurate assessment (13). Second, LGE images were used as reference to detect the infarcted myocardium and MV0 on T1 maps. To evaluate the diagnostic performance of T1 maps for detecting and delineating MVO compared to that of LGE images, the operators should be blinded to LGE images when they were analyzing T1 maps. Third, histopathological corroboration of the infarcted myocardium, MV0, and remote myocardium was not performed. Ideally, the tissues that correspond to the measured areas on images should be observed in order to help identify mechanisms that may have led to T1 value changes. Fourth, $2^{*}$-weighted CMR was not performed to detect myocardial hemorrhage. Finally, this was a retrospective, single-center study with a small sample size, and thus the findings may not be generalizable to all patients. Therefore, studies with larger sample sizes are necessary for further validation.

In conclusion herein, using native T1 maps, MV0 areas were accurately delineated and these areas showed high concordance with the areas measured on LGE images. However, post-contrast T1 maps had low MVO detection rates and produced underestimated MVO areas. Collectively, 
our findings suggest that native T1 mapping is a useful tool for detecting MVO within an infarcted myocardium that clearly shows a low $\mathrm{T} 1$ value.

\section{Conflicts of Interest}

The authors have no potential conflicts of interest to disclose.

\author{
ORCID iDs \\ Tae Hoon Kim \\ https://orcid.org/0000-0003-3598-2529 \\ Jae Min Shin \\ https://orcid.org/0000-0003-4549-3518 \\ Eui-Young Choi \\ https://orcid.org/0000-0003-3732-0190 \\ Chul Hwan Park \\ https://orcid.org/0000-0002-0004-9475 \\ Kyunghwa Han \\ https://orcid.org/0000-0002-5687-7237
}

\section{REFERENCES}

1. Azarisman SM, Carbone A, Shirazi M, Bradley J, Teo KS, Worthley MI, et al. Characterisation of myocardial injury via t1 mapping in early reperfused myocardial infarction and its relationship with global and regional diastolic dysfunction. Heart Lung Circ 2016;25:1094-1106

2. Varga-Szemes $A$, van der Geest RJ, Spottiswoode BS, Suranyi $P$, Ruzsics B, De Cecco CN, et al. Myocardial late gadolinium enhancement: accuracy of T1 mapping-based synthetic inversion-recovery imaging. Radiology 2016;278:374-382

3. Nezafat R. Native T1 mapping for myocardial infarction: time to throw out the gadolinium? JACC Cardiovasc Imaging 2015;8:1031-1033

4. Abbas A, Matthews GH, Brown IW, Shambrook JS, Peebles CR, Harden SP. Cardiac MR assessment of microvascular obstruction. Br J Radiol 2015;88:20140470

5. Biesbroek PS, Amier RP, Teunissen PFA, Hofman MBM, Robbers LFHJ, van de Ven PM, et al. Changes in remote myocardial tissue after acute myocardial infarction and its relation to cardiac remodeling: a CMR T1 mapping study. PLoS One 2017;12:e0180115

6. Doltra A, Amundsen BH, Gebker R, Fleck E, Kelle S. Emerging concepts for myocardial late gadolinium enhancement MRI. Curr Cardiol Rev 2013;9:185-190

7. Wu KC. CMR of microvascular obstruction and hemorrhage in myocardial infarction. J Cardiovasc Magn Reson 2012;14:68

8. Rogosnitzky M, Branch S. Gadolinium-based contrast agent toxicity: a review of known and proposed mechanisms. Biometals 2016;29:365-376

9. Dall'Armellina E, Piechnik SK, Ferreira VM, Si QL, Robson MD,
Francis JM, et al. Cardiovascular magnetic resonance by non contrast T1-mapping allows assessment of severity of injury in acute myocardial infarction. J Cardiovasc Magn Reson 2012;14:15

10. Robbers LFHJ, Nijveldt R, Beek AM, Teunissen PFA, Hollander $M R$, Biesbroek PS, et al. The influence of microvascular injury on native $\mathrm{T} 1$ and $\mathrm{T} 2$ * relaxation values after acute myocardial infarction: implications for non-contrast-enhanced infarct assessment. Eur Radiol 2018;28:824-832

11. Cameron D, Siddiqi N, Neil CJ, Jagpal B, Bruce M, Higgins $D M$, et al. $T_{1}$ mapping for assessment of myocardial injury and microvascular obstruction at one week post myocardial infarction. Eur J Radiol 2016;85:279-285

12. Garg P, Saunders LC, Swift AJ, Wild JM, Plein S. Role of cardiac T1 mapping and extracellular volume in the assessment of myocardial infarction. Anatol J Cardiol 2018;19:404-411

13. Kellman P, Hansen MS. T1-mapping in the heart: accuracy and precision. J Cardiovasc Magn Reson 2014;16:2

14. Kim PK, Hong YJ, Im DJ, Suh YJ, Park CH, Kim JY, et al. Myocardial T1 and T2 mapping: techniques and clinical applications. Korean J Radiol 2017;18:113-131

15. Dastidar AG, Harries I, Pontecorboli G, Bruno VD, De Garate E, Moret $C$, et al. Native T1 mapping to detect extent of acute and chronic myocardial infarction: comparison with late gadolinium enhancement technique. Int J Cardiovasc Imaging 2019;35:517-527

16. Bulluck H, Rosmini S, Abdel-Gadir A, Bhuva AN, Treibel TA, Fontana M, et al. Diagnostic performance of T1 and T2 mapping to detect intramyocardial hemorrhage in reperfused ST-segment elevation myocardial infarction (STEMI) patients. J Magn Reson Imaging 2017;46:877-886

17. Bulluck H, Hammond-Haley M, Fontana M, Knight DS, Sirker A, Herrey AS, et al. Quantification of both the area-at-risk and acute myocardial infarct size in ST-segment elevation myocardial infarction using T1-mapping. J Cardiovasc Magn Reson 2017;19:57

18. Mather AN, Lockie T, Nagel E, Marber M, Perera D, Redwood $S$, et al. Appearance of microvascular obstruction on high resolution first-pass perfusion, early and late gadolinium enhancement CMR in patients with acute myocardial infarction. J Cardiovasc Magn Reson 2009;11:33

19. Liu D, Borlotti A, Viliani D, Jerosch-Herold M, Alkhalil M, De Maria GL, et al. CMR native T1 mapping allows differentiation of reversible versus irreversible myocardial damage in STsegment-elevation myocardial infarction: an 0xAMI study (0xford Acute Myocardial Infarction). Circ Cardiovasc Imaging 2017;10:e005986

20. Piechnik SK, Ferreira VM, Dall'Armellina E, Cochlin LE, Greiser A, Neubauer S, et al. Shortened modified look-locker inversion recovery (ShMOLLI) for clinical myocardial T1-mapping at 1.5 and $3 \mathrm{~T}$ within a 9 heartbeat breathhold. J Cardiovasc Magn Reson 2010;12:69

21. Heuschmid M, Rothfuss JK, Schroeder S, Fenchel M, Stauder N, 
Burgstahler $C$, et al. Assessment of left ventricular myocardial function using 16-slice multidetector-row computed tomography: comparison with magnetic resonance imaging and echocardiography. Eur Radiol 2006;16:551-559

22. Grothues F, Smith GC, Moon JC, Bellenger NG, Collins P, Klein $\mathrm{HU}$, et al. Comparison of interstudy reproducibility of cardiovascular magnetic resonance with two-dimensional echocardiography in normal subjects and in patients with heart failure or left ventricular hypertrophy. Am J Cardiol 2002;90:29-34

23. Messroghli DR, Moon JC, Ferreira VM, Grosse-Wortmann L, He T, Kellman $\mathrm{P}$, et al. Clinical recommendations for cardiovascular magnetic resonance mapping of $\mathrm{T} 1, \mathrm{~T} 2, \mathrm{~T} 2$ * and extracellular volume: a consensus statement by the Society for Cardiovascular Magnetic Resonance (SCMR) endorsed by the European Association for Cardiovascular Imaging (EACVI). J Cardiovasc Magn Reson 2017;19:75

24. Williams ES, Kaplan JI, Thatcher F, Zimmerman G, Knoebel $\mathrm{SB}$. Prolongation of proton spin lattice relaxation times in regionally ischemic tissue from dog hearts. J Nucl Med 1980;21:449-453
25. Ghugre NR, Pop M, Barry J, Connelly KA, Wright GA. Quantitative magnetic resonance imaging can distinguish remodeling mechanisms after acute myocardial infarction based on the severity of ischemic insult. Magn Reson Med 2013;70:1095-1105

26. Kali A, Kumar A, Cokic I, Tang RL, Tsaftaris SA, Friedrich $M G$, et al. Chronic manifestation of postreperfusion intramyocardial hemorrhage as regional iron deposition: a cardiovascular magnetic resonance study with ex vivo validation. Circ Cardiovasc Imaging 2013;6:218-228

27. Ghugre NR, Wood JC. Relaxivity-iron calibration in hepatic iron overload: probing underlying biophysical mechanisms using a Monte Carlo model. Magn Reson Med 2011;65:837-847

28. Wu KC, Zerhouni EA, Judd RM, Lugo-Olivieri CH, Barouch LA, Schulman SP, et al. Prognostic significance of microvascular obstruction by magnetic resonance imaging in patients with acute myocardial infarction. Circulation 1998;97:765-772

29. Mather AN, Fairbairn TA, Ball SG, Greenwood JP, Plein S. Reperfusion haemorrhage as determined by cardiovascular MRI is a predictor of adverse left ventricular remodelling and markers of late arrhythmic risk. Heart 2011;97:453-459 